\title{
Fluctuations of a passive scalar in a turbulent mixing layer
}

\author{
Antonio Attili ${ }^{*}$ and Fabrizio Bisetti \\ Clean Combustion Research Center, King Abdullah University of Science and Technology, Thuwal 23955, Kingdom of Saudi Arabia
}

(Received 7 September 2012; revised manuscript received 9 July 2013; published 19 September 2013)

\begin{abstract}
The turbulent flow originating downstream of the Kelvin-Helmholtz instability in a mixing layer has great relevance in many applications, ranging from atmospheric physics to combustion in technical devices. The mixing of a substance by the turbulent velocity field is usually involved. In this paper, a detailed statistical analysis of fluctuations of a passive scalar in the fully developed region of a turbulent mixing layer from a direct numerical simulation is presented. Passive scalar spectra show inertial ranges characterized by scaling exponents $-4 / 3$ and $-3 / 2$ in the streamwise and spanwise directions, in agreement with a recent theoretical analysis of passive scalar scaling in shear flows [Celani et al., J. Fluid Mech. 523, 99 (2005)]. Scaling exponents of high-order structure functions in the streamwise direction show saturation of intermittency with an asymptotic exponent $\zeta_{\infty}=0.4$ at large orders. Saturation of intermittency is confirmed by the self-similarity of the tails of the probability density functions of the scalar increments at different scales $r$ with the scaling factor $r^{-\zeta_{\infty}}$ and by the analysis of the cumulative probability of large fluctuations. Conversely, intermittency saturation is not observed for the spanwise increments and the relative scaling exponents agree with recent results for homogeneous isotropic turbulence with mean scalar gradient. Probability density functions of the scalar increments in the three directions are compared to assess anisotropy.
\end{abstract}

DOI: 10.1103/PhysRevE.88.033013

PACS number(s): 47.27.-i, 47.51.+a

\section{INTRODUCTION}

The advection of a substance by a turbulent velocity field is a fundamental process in nature and many engineering applications [1,2]. Examples include moisture and pollutants in the atmosphere or chemical species in a combustion chamber. If the substance does not affect the dynamics of the velocity field, the substance is referred to as a passive scalar.

The turbulent velocity field folds and stretches the trajectories of fluid parcels, inducing large scalar gradients. The net effect is a significant increase in scalar mixing (the dissipation of the scalar variance) associated with large fluctuations in the spatiotemporal scalar field.

From a practical point of view, large fluctuations and extreme events play a critical role. For example, the probability of exceeding a certain concentration threshold is of primary importance in the dispersion of a pollutant or the ignition of a nonhomogeneous mixture [3]. Moreover, phenomena relevant in applications such as mixing and reaction occur at the smallest scales, so engineering models cannot ignore the statistics and morphology of the scalar field at these scales where the strongest deviations from Gaussian behavior are observed [2]. Examples of the strong effects that the non-Gaussian and intermittent scalar field has on quantities of practical relevance are reported by Sreenivasan [4], who describes how intermittency in the scalar dissipation rate affects reactive turbulent flow physics and modeling, and Edouard et al. [5], who provide an example of how small-scale inhomogeneities control the overall rate of depletion of ozone in the Arctic region.

In real flows, turbulence is often generated by instabilities and mean flow shear is important. The turbulent mixing

*antonio.attili@kaust.edu.sa layer is one of the simplest canonical flow characterized by shear, instability, and large coherent structures. The large-scale motion of the flow is dominated by quasi-two-dimensional structures, described for the first time in the seminal work of Brown and Roshko [6]. These structures originate from the roll-up of the vortices generated by the Kelvin-Helmholtz instability [7] and persist in the far field, where well-developed turbulence is usually observed [8]. Significant efforts have been directed toward the analysis of the mixing layer by theoretical $[9,10]$, experimental $[6,11,12]$, and numerical $[7,13-18]$ means in order to characterize the dynamics of large organized structures $[6,7,13,14]$, the process of the transition to turbulence $[7,16]$, the self-similar state $[11,12,15]$, and the mixing layer's nonuniversal nature [16-18]. By contrast, a limited number of studies describe the statistical features of fluctuations in the mixing layer turbulence [8,19-21] and a detailed statistical analysis of passive scalar fluctuations in a turbulent mixing layer is lacking.

It is well known [1,2] that high-order statistics of passive scalar turbulence are characterized by strong deviations from predictions based on classical dimensional scaling arguments proposed by Corrsin [22] and Obukhov [23]. Like velocity, scalar transport is dominated by intermittency. This is apparent in both the scale dependence of the probability density functions (PDFs) of the scalar increments and the deviation of the high-order structure functions scaling from dimensional predictions. The so-called anomalous scaling of the scalar is more pronounced than that observed for velocity. This is not a consequence of the intermittency of the velocity field since the anomalous scaling of the scalar is observed for nonintermittent velocity fields also [24-27]. The statistical behavior of the scalar field is explained by the appearance of frontlike structures [28,29]. These fronts occur frequently, causing anomalous scaling and compromising isotropy recovery, possibly down to the smallest scales [30]. The quasidiscontinuous nature of these scalar fronts leads 
to saturation of intermittency [28,31-33], i.e., the scaling exponents of the structure function reach an asymptotic value as the order increases.

Even though intermittency is expected to have limited effects on the scalar spectrum and the second-order structure function, significant deviations from the classical ObukhovCorrsin $-5 / 3$ scaling [22,23] have been reported for these low-order statistics. In the case of shear flows, the deviations are quite large and apparent even when the velocity spectrum is characterized by a well-defined inertial range with a scaling exponent very close to $-5 / 3$ [2,34-36]. Only at very large Reynolds numbers $\left(\operatorname{Re}_{\lambda} \geqslant 2000\right)$ is the $-5 / 3$ scaling recovered. Recently, Celani et al. [37] proposed a theoretical argument, based on Lagrangian dynamics, to explain the scaling of the passive scalar spectra observed in shear turbulence. The presence of shear introduces a new length scale $L_{s}$ [38]. At scales larger than $L_{s}$ and assuming constant shear, Celani et al. [37] calculate spectra scaling exponents of $-4 / 3$ and $-3 / 2$ for the directions parallel and perpendicular to the mean shear, respectively.

Given the relevance of the configuration and the limited literature on the subject, it is of great interest to characterize the role of mean shear and coherent large structures in the statistics of passive scalar fluctuations in the turbulent mixing layer. After a brief description of the configuration and methods (Sec. II), the scaling of second-order statistics, i.e., spectra and second-order structure functions, is analyzed and compared to theoretical predictions for constant shear flows [37] (Sec. III). In Sec. IV, high-order statistics are reported and intermittency saturation is assessed using scaling of structure functions. Moreover, the PDFs of scalar increments in the three directions are analyzed to confirm the presence of intermittency saturation and to evaluate the anisotropy of the scalar fields.

\section{PRELIMINARIES}

The direct numerical simulation (DNS) presented in this work is performed by solving the unsteady, incompressible Navier-Stokes equations. An additional transport equation for a passive scalar $\Phi$ with Schmidt number equal to 0.7 is also solved.

The parallel flow solver NGA [39] developed at Stanford University is used to solve the transport equations. The solver implements a finite-difference method on a spatially and temporally staggered grid with the semi-implicit fractionalstep method of Kim and Moin [40]. Velocity and scalar spatial derivatives are discretized with a second-order finitedifference-centered scheme.

A complete description of the flow parameters and methods used for the computation is reported in [8], together with a detailed analysis of the spatial evolution of the flow and velocity statistics in the transitional and fully developed turbulent regions. Therefore, only a brief summary is reported here.

A schematic description of the geometry is shown in Fig. 1. The flow is imposed at the inlet plane $(x=0)$ and free convective outflow [41] is specified at $x=L_{x}$. The boundary conditions are periodic in the spanwise direction $z$ and free slip in the crosswise direction $y$. The flow at the inlet $(x=0)$

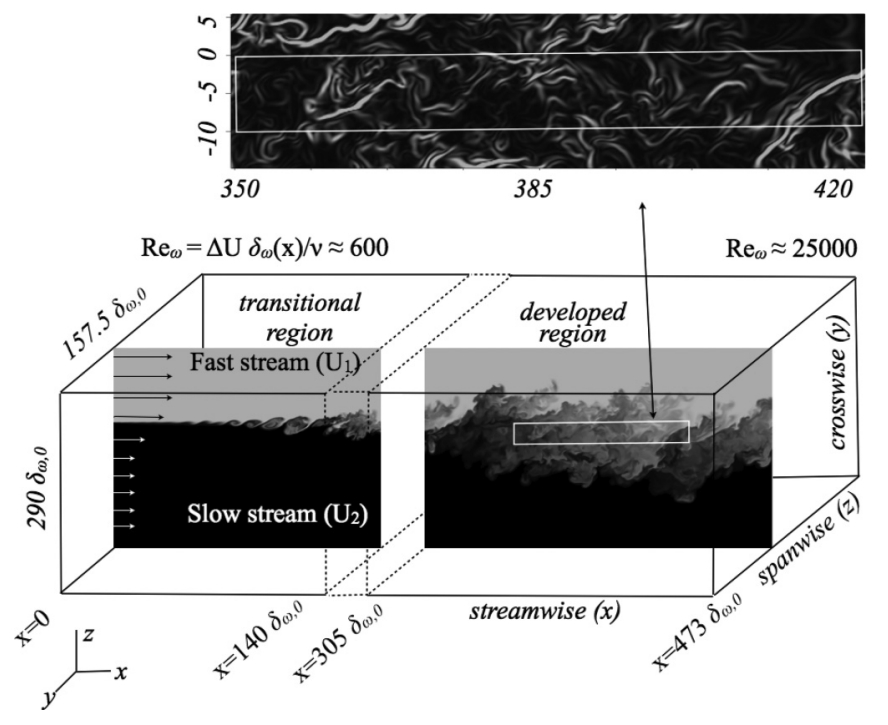

FIG. 1. Schematic description of the flow configuration. The inlet is on the left $(x=0)$ and the outlet on the right $\left(x=473 \delta_{\omega, 0}\right)$. The statistical analysis of scalar fluctuations was performed in the region marked by the white boxes: $350 \delta_{\omega, 0}<x<425 \delta_{\omega, 0}$ and $y=-9 \delta_{\omega, 0}<y<-\delta_{\omega, 0}$. A two-dimensional cut of the scalar field, ranging from $\Phi=\Phi_{2}$ (dark gray) to $\Phi=\Phi_{1}$ (light gray), is shown in the bottom figure. The top figure shows an enlarged view of the squared gradient of the scalar field (scalar dissipation) in the region analyzed. Light (dark) gray corresponds to large (small) values of the gradient.

is a hyperbolic tangent profile for the streamwise velocity $U$ with prescribed vorticity thickness $\delta_{\omega, 0}$ :

$$
U(x=0, y, z)=U_{c}+\frac{1}{2} \Delta U \tanh \left(\frac{2 y}{\delta_{\omega, 0}}\right),
$$

where $U_{c}=\left(U_{1}+U_{2}\right) / 2$ is the convective velocity, $U_{1}$ and $U_{2}$ are the high- and low-speed stream velocities, and $\Delta U=U_{1}-U_{2}$ is the velocity difference across the layer. The Reynolds number based on the vorticity (momentum) thickness at the inlet is $\operatorname{Re}_{\omega}=600\left(\operatorname{Re}_{\theta}=150\right)$, increasing up to $\operatorname{Re}_{\omega}=25000\left(\operatorname{Re}_{\theta}=4250\right)$ as the mixing layer develops. The ratio of the two velocities is $U_{1} / U_{2}=3$. Lowamplitude white noise is superimposed on the hyperbolic tangent profile, resulting in the onset of the Kelvin-Helmholtz instability at a short distance downstream of the inlet $(x \approx$ $\left.50 \delta_{\omega, 0}\right)$. The crosswise and spanwise velocity components are perturbed with the same type of disturbance. At the inlet boundary, the passive scalar is $\Phi_{1}$ and $\Phi_{2}$ in the highand low-speed streams, respectively, with a smooth transition between the two values.

The computational domain extends over $L_{x}=473 \delta_{\omega, 0}$, $L_{y}=290 \delta_{\omega, 0}$, and $L_{z}=157.5 \delta_{\omega, 0}$ in the streamwise $x$, crosswise $y$, and spanwise $z$ directions, respectively. The domain is discretized with $3072 \times 940 \times 1024 \approx 3 \times 10^{9}$ grid points $\left(N_{x} \times N_{y} \times N_{z}\right)$. In the region centered around $y=0$ $\left(|y| \leqslant 45 \delta_{\omega, 0}\right)$, the grid is homogeneous in the three directions: $\Delta x=\Delta y=\Delta z=0.15 \delta_{\omega, 0}$. Outside the core region for $|y|>$ $45 \delta_{\omega, 0}$, the grid is stretched linearly up to $\Delta y=0.6 \delta_{\omega, 0}$ at $|y|=55 \delta_{\omega, 0}$ and is homogeneous up to the boundary. In the region $45 \delta_{\omega, 0}<|y|<55 \delta_{\omega, 0}$, the vorticity and the fluctuating 
vorticity root mean square are respectively three and five orders of magnitude smaller than inside the layer, meaning that the turbulent flow never reaches $|y|=45 \delta_{\omega, 0}$. Overall, the spatial resolution is such that $\Delta x=\Delta y=\Delta z \leqslant 2.5 \eta$ everywhere, where $\eta=v^{3 / 4} \varepsilon^{-1 / 4}$ is the Kolmogorov scale and $\varepsilon$ the turbulent kinetic energy dissipation rate. The time step size is calculated in order to have a unity Courant-Friedrichs-Lewy number.

The simulation was performed on the IBM Blue Gene/P system Shaheen available at King Abdullah University of Science and Technology, using up to 65536 processing cores (16 racks of the Blue Gene/P architecture). Statistics were accumulated over time for $3500 \tau\left(\tau=\Delta U / \delta_{\omega, 0}\right)$ and 1400 flow field samples were used to evaluate statistics. Several time signals were sampled at various spatial locations to complement the spatial statistics with their temporal surrogates by Taylor's hypothesis. The simulation required around $10 \times 10^{6} \mathrm{CPU}$ hours and produced approximately $100 \mathrm{~TB}$ of data.

It is well known that at a certain distance from the inlet, the mixing layer evolves self-similarly. Appropriate scaling velocity and length scales are the constant velocity difference $\Delta U$ across the mixing layer and a measure of the local layer thickness, e.g., momentum or vorticity thickness $[42,43]$. In a previous analysis of the same DNS database [8], it was shown that the local layer thickness and the mean and variance of velocity evolve self-similarly for $x>300 \delta_{\omega, 0}$.

The analysis presented in this paper was performed in the self-similar region between $x=350 \delta_{\omega, 0}$ and $425 \delta_{\omega, 0}$ and between $y=-9 \delta_{\omega, 0}$ and $-\delta_{\omega, 0}$, near the crosswise location of maximum velocity variance (see Fig. 1). Figure 2 shows the crosswise profiles of the mean and variance of velocity and passive scalar at $x=383 \delta_{\omega, 0}$. Angular brackets indicate a statistical mean obtained by averaging in the spanwise direction $z$ and time $t$ and $u=U-\langle U\rangle, v=V-\langle V\rangle$, and $w=W-\langle W\rangle$ are the velocity fluctuations in the three directions. Analogously, $\phi=\Phi-\langle\Phi\rangle$ indicates the fluctuations of

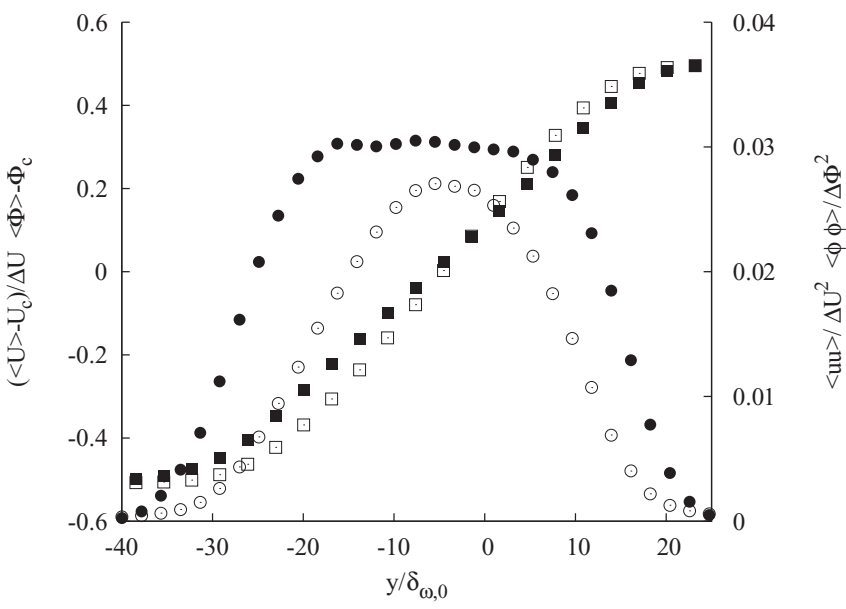

FIG. 2. Mean velocity $\left(\langle U\rangle-U_{c}\right) / \Delta U$ (open squares) scaled with the convective velocity $U_{c}=\left(U_{1}+U_{2}\right) / 2$ and velocity difference $\Delta U=U_{1}-U_{2}$, velocity fluctuation variance $\langle u u\rangle / \Delta U^{2}$ (open circles), mean passive scalar $\left(\langle\Phi\rangle-\Phi_{c}\right) / \Delta \Phi$ (closed squares) scaled with $\Phi_{c}=\left(\Phi_{1}+\Phi_{2}\right) / 2$ and $\Delta \Phi=\Phi_{1}-\Phi_{2}$, and passive scalar fluctuation variance $\sigma^{2}=\langle\phi \phi\rangle / \Delta \Phi^{2}$ (closed circles).
TABLE I. Flow parameters in the region used for the analysis $\left(x=350 \delta_{\omega, 0}\right.$ and $\left.425 \delta_{\omega, 0}\right)$. The values of the Kolmogorov scale, production, and dissipation are calculated at the crosswise position of maximum turbulent kinetic energy $\left(y \approx-5 \delta_{\omega, 0}\right)$. Here $L_{s}=\sqrt{\varepsilon / \mathcal{S}^{3}}$ is the shear length scale, $\mathcal{S}$ being the local mean shear and $\varepsilon$ the dissipation.

\begin{tabular}{lcc}
\hline Kolmogorov scale & $\eta$ & $0.07 \delta_{\omega, 0}$ \\
turbulence production/dissipation ratio & $P / \varepsilon$ & 1.4 \\
resolution & $\Delta x / \eta$ & 2 \\
shear length scale & $L_{s}$ & $\approx 40 \eta$ \\
vorticity thickness & $\delta_{\omega}$ & $\approx 500 \eta$ \\
Taylor microscale & $\lambda_{T}$ & $\approx 43 \eta$ \\
Reynolds number (Taylor microscale) & $\mathrm{Re}_{\lambda}$ & $\approx 250$ \\
\hline
\end{tabular}

the scalar field $\Phi$ with respect to its mean $\langle\Phi\rangle$ and $\sigma$ is the root mean square (rms). In the region analyzed, the streamwise and crosswise variations of mean shear, mean scalar gradient, and velocity and scalar variance are less than $5 \%$. Table I summarizes some important flow parameters in this region.

\section{SECOND-ORDER STATISTICS}

\section{A. Shear effects on passive scalar spectra}

In the presence of mean shear, an additional length scale $L_{s}=\sqrt{\varepsilon / \mathcal{S}^{3}}$ may be introduced, where $\mathcal{S}$ is the local mean shear and $\varepsilon$ the dissipation [38,44,45]. This scale identifies the cutoff between the small scales, which are not affected by mean shear and display homogeneous isotropic turbulence scaling, and larger scales dominated by mean shear, showing different scaling properties (see [44] for a detailed analysis of the velocity scaling in shear flows).

The effect of mean shear on the scaling of second-order statistics for the passive scalar is described by Celani et al. $[37,46]$ using Lagrangian dynamics [47]. A brief summary of the derivation is reported here for clarity.

The second-order structure function for the scalar is defined at position $\rho=\{x, y, z\}$ as

$$
S_{2}(\boldsymbol{\rho}, \mathbf{r})=\left\langle[\phi(\rho+\mathbf{r})-\phi(\rho)]^{2}\right\rangle,
$$

where $\mathbf{r}$ is a spatial separation and angular brackets denote an appropriate statistical average. The scaling of the spectrum $E(k)$ versus $k$ is related to the scaling of the second-order structure function with respect to the separation $r=|\mathbf{r}|$ [48]: The scaling $E(k) \propto k^{-\alpha}$ corresponds to $S_{2}(r) \propto r^{\alpha-1}$. The structure function $S_{2}(r)$ has a straightforward Lagrangian interpretation: Its value is proportional to the time $\mathcal{T}(r)$ required for two particles, initially at the same position, to separate by a distance $r$ [47]. For simple flow configurations, it is possible to estimate the scaling of $\mathcal{T}(r)$ so that the spectrum exponent $\alpha$ can be calculated. In the case of constant shear, Celani et al. [37] provide a quantitative estimation of the spectra scaling in the direction parallel and perpendicular to the shear.

First, a simple model for the velocity advecting the passive scalar is assumed:

$$
\mathbf{v}(\rho, t)=\mathcal{S} y \hat{\mathbf{x}}+\mathbf{u}(\rho, t) .
$$


This velocity field is a combination of a linear shear with constant intensity $\mathcal{S}$ and a three-dimensional isotropic field obeying Kolmogorov's $k^{-5 / 3}$ scaling. The evolution of the separation between two particles is given by

$$
\begin{gathered}
\dot{R}_{x}=\mathcal{S} R_{y}+\delta u_{x}(\mathbf{R}, t), \\
\dot{\mathbf{R}}_{\perp}=\delta \mathbf{u}_{\perp}(\mathbf{R}, t) .
\end{gathered}
$$

The overdot indicates a time derivative, $\delta \mathbf{u}$ is the velocity difference between two particles, and $R_{x}$ and $\mathbf{R}_{\perp}=\left\{R_{y}, R_{z}\right\}$ are the components of the particles' separation in the directions parallel and perpendicular to the mean shear. At scales smaller than $L_{s}$, the dynamics are dominated by the turbulent component $\delta \mathbf{u}$ and the effects of the shear term $\mathcal{S} R_{y}$ are negligible. In this limit, $\delta \mathbf{u}$ scales with exponent $1 / 3$ due to classical Kolmogorov scaling and Richardson's law holds: $\left\langle R^{2}(t)\right\rangle \propto t^{3}$, or equivalently $\mathcal{T}(r) \propto r^{2 / 3}$. Hence the scalings $r^{2 / 3}$ and $k^{-5 / 3}$ are recovered for the second-order structure function and scalar spectrum. At scales larger than $L_{S}$, or equivalently for times greater than $t_{s}$, defined as the time required for two particles to reach a separation comparable to $L_{s}$, the dynamics are dominated by the term $\mathcal{S} R_{y}$. Therefore, $R_{x}$ and $\mathbf{R}_{\perp}$ show a different scaling, i.e., $\left\langle R_{x}^{2}\right\rangle \approx\left\langle R^{2}\right\rangle \propto t^{2 a}$ and $\left\langle R_{\perp}^{2}\right\rangle \propto t^{2 b}$, respectively. Invoking Kolmogorov's scaling $\delta \mathbf{u}_{\perp}(\mathbf{R}, t) \propto R^{1 / 3}$ and neglecting $\delta u_{x}(\mathbf{R}, t)$, the two relations $a-1=b$ and $b-1=a / 3$ are obtained. Hence the following scalings apply:

$$
S_{x, 2} \propto r^{1 / 3}, \quad E_{x}(k) \propto k^{-4 / 3}
$$

and

$$
S_{\perp, 2} \propto r^{1 / 2}, \quad E_{\perp}(k) \propto k^{-3 / 2},
$$

where $x$ and $\perp$ indicate the directions parallel and perpendicular to the mean shear.

\section{B. Passive scalar spectra and second-order structure function in the turbulent mixing layer}

Spectra and second-order structure functions for the passive scalar were calculated in the self-similar region of the turbulent mixing layer for the streamwise $x$ and spanwise $z$ directions, which are respectively parallel and perpendicular to the mean shear. The flow is highly nonhomogeneous in the crosswise direction, therefore the spectrum and structure functions have not been calculated in this direction. It is worth noting that in a previous work using the same database [8], it has been shown that velocity spectra in the fully turbulent region show a wide inertial range with $k^{-5 / 3}$ scaling and, at scales smaller than $250 \eta$, the structure functions are similar to those in homogeneous isotropic turbulence at a comparable Reynolds number.

Passive scalar spectra are shown in Fig. 3. The spectra in both the $x$ and $z$ directions show an inertial range with constant scaling. The compensated spectra, shown in the inset, illustrate that appropriate scalings are $k^{-4 / 3}$ and $k^{-3 / 2}$ for the $x$ and $z$ directions, in agreement with the theoretical analysis of Celani et al. [37] summarized in Eqs. (6) and (7). It is also evident that the scaling $k^{-5 / 3}$ is not appropriate to describe the inertial range for either spectrum. In the theoretical analysis it is assumed that the mean shear is constant in space and time. In a mixing layer,

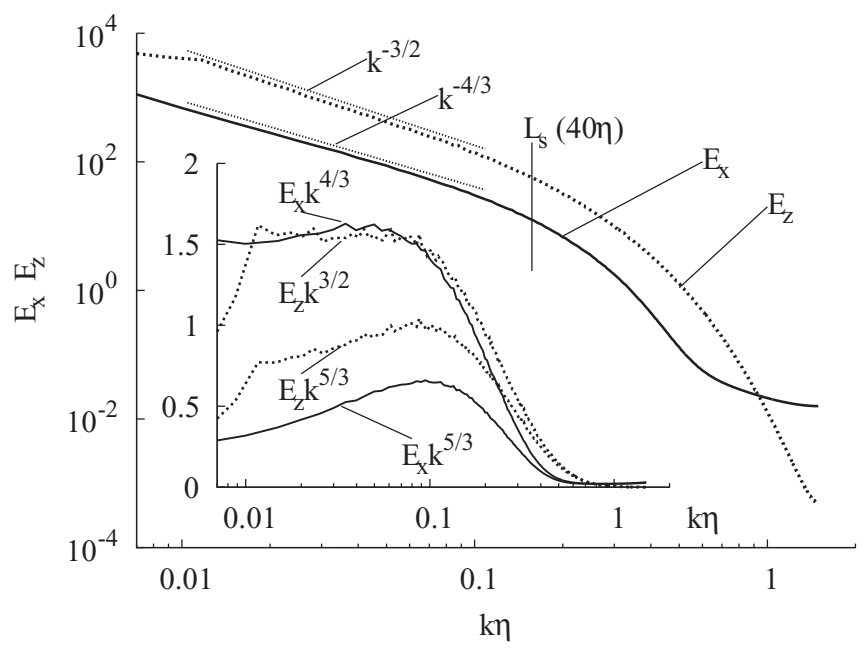

FIG. 3. Passive scalar spectra in the streamwise $x$ (solid lines) and spanwise $z$ (dashed lines) directions. The straight dotted lines represent the predictions of equations (6) and (7). The vertical straight line indicates the shear length scale $L_{s} \approx 40 \eta$. The inset shows the streamwise and spanwise spectra multiplied by $k^{4 / 3}$ and $k^{3 / 2}$, respectively. The spectra compensated by the classical scaling $k^{-5 / 3}$ are also shown for comparison.

mean shear is constant in time but varies in the streamwise and crosswise directions. In the region considered, the variations of mean shear and relevant turbulent quantities are small (Fig. 2). Therefore, the hypotheses for which the scalings $k^{-4 / 3}$ and $k^{-3 / 2}$ hold are satisfied. The present configuration does not allow for investigating the recovery of the classical $k^{-5 / 3}$ scaling at scales smaller than $L_{s}$ because $L_{s} \approx 40 \eta$, so the entire inertial range is dominated by shear.

Figure 4 show the second-order structure function in the streamwise and spanwise directions. The insets show the logarithmic derivative, corresponding to the local slope in a $\log$-log plot. At scales of the order of the Kolmogorov length, the viscous scaling is recovered and the structure functions are proportional to $r^{2}$. As it is usually observed in turbulent fields, the inertial range in the second-order structure function appears to be smaller than in the spectrum and a constant scaling range is not evident. Nevertheless, it is apparent that, for the streamwise direction, the exponent $2 / 3$, which corresponds to the classical spectrum scaling $k^{-5 / 3}$, is rather unlikely, while the value $1 / 3$ may better describe the structure function. In the spanwise direction, the range of scales with an approximately constant scaling exponent is even smaller. In order to identify scaling ranges for the analysis to follow, a procedure analogous to the one used by and Anselmet et al. [49] and Lepore and Mydlarski [50] has been employed. The upper and lower bounds of the scaling range are defined by the locations at which the compensated second-order structure functions fall to $90 \%$ of their maximum value. In this approach, the structure functions are compensated by the scaling observed for the spectra, in agreement with the theory by Celani et al. [37] for shear flows. These bounds are reported in the insets of Fig. 4. The scaling ranges are $120 \eta<r<280 \eta$ and $100 \eta<r<200 \eta$ for the streamwise and spanwise directions, respectively. Differently from the procedure used here, Lepore and Mydlarski [50] employ the compensated mixed third-order 

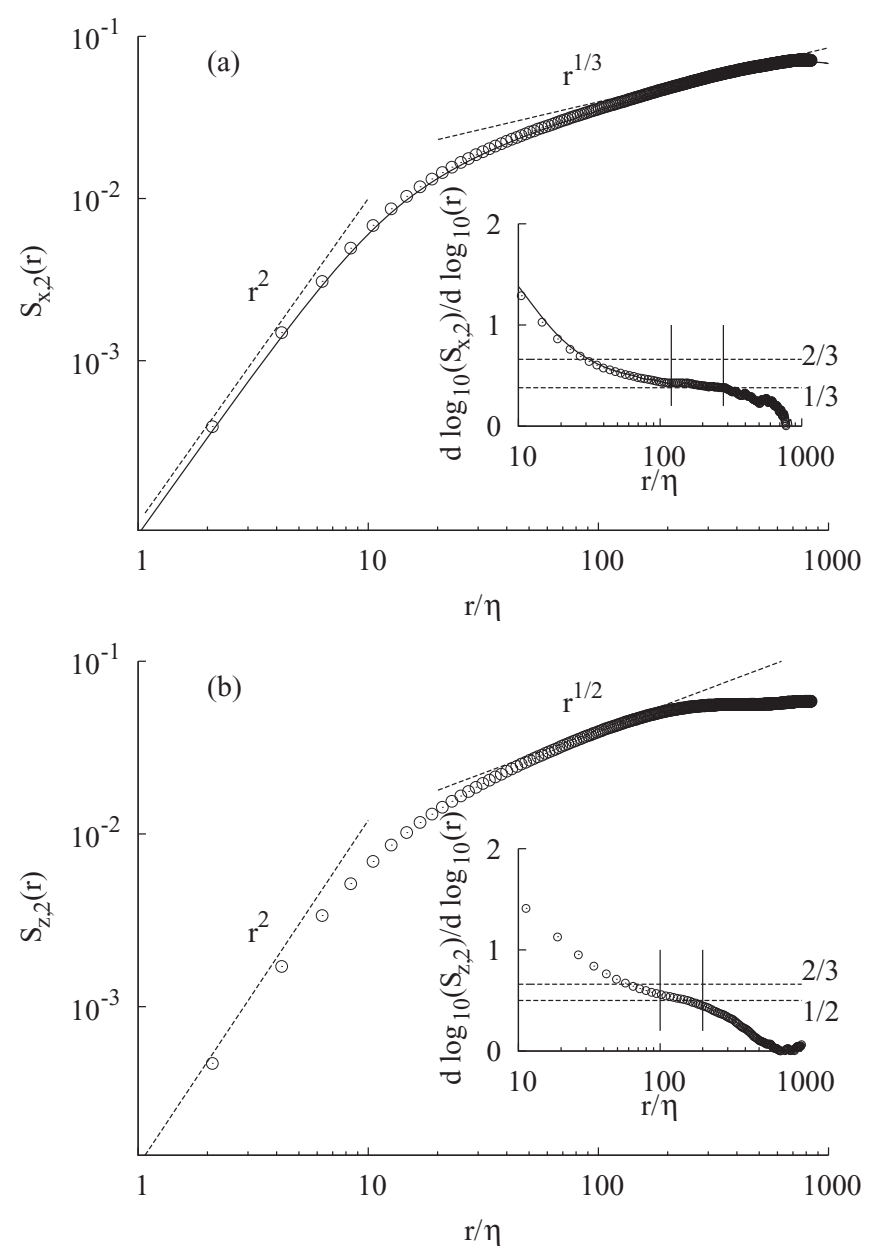

FIG. 4. Second-order structure function (circles) in the (a) streamwise $x$ and (b) spanwise $z$ directions. In the $x$ direction, the structure function calculated using time signals and Taylor's hypothesis is also shown (solid line). The dashed lines show the scaling in the viscous and inertial ranges. The insets show the logarithmic derivative of the structure function with respect to separation. The dashed lines indicate the Kolmogorov-Obukhov-Corrsin dimensional scaling $(2 / 3)$ and the anomalous scaling exponents $(1 / 3$ and $1 / 2$ ) consistent with those of the spectra. The vertical solid lines mark the regions with scaling exponent in agreement with the spectra.

structure function because the statistic are not affected by intermittency and, at least in the homogeneous and isotropic case, are expected to scale according to the dimensional prediction [22,23]. In the present case dominated by mean shear, the dimensional prediction may be invalid. Therefore, the second-order structure functions have been preferred given the availability of a theoretical prediction for their scaling in flows with mean shear [37].

For the streamwise direction, the second-order structure function obtained from temporal surrogates via Taylor's hypothesis is also shown. The temporal surrogate is obtained by sampling the passive scalar field in time at an array of 128 spatially distributed points. The probes are distributed across the spanwise $z$ direction at $x=383 \delta_{\omega, 0}$ and $y=$ $-5 \delta_{\omega, 0}$. The temporal and spatial statistics are in excellent agreement.

\section{HIGH-ORDER STATISTICS AND SATURATION OF INTERMITTENCY}

The statistical properties of turbulent fluctuations in the inertial range can be characterized in terms of the scaling of high-order structure functions [48,51]. The $n$ th-order structure function for the passive scalar $\phi$ is defined as

$$
S_{n}(\boldsymbol{\rho}, \mathbf{r})=\left\langle\delta_{\mathbf{r}} \phi(\boldsymbol{\rho})\right\rangle=\left\langle[\phi(\boldsymbol{\rho}+\mathbf{r})-\phi(\boldsymbol{\rho})]^{n}\right\rangle,
$$

where $\delta_{\mathbf{r}} \phi(\rho)$ is the scalar increment at position $\rho=$ $\{x, y, z\}$ over the spatial separation $\mathbf{r}$. For even-order structure functions, classical theoretical arguments for homogeneous isotropic turbulence [22,23] predict

$$
S_{n}(r) \propto r^{\zeta_{n}}
$$

with $\zeta_{n}=n / 3$. In the isotropic case, odd-order structure functions vanish by symmetry. In the presence of a mean scalar gradient, dimensional arguments give, for odd orders,

$$
S_{2 n+1}(r) \propto r^{\zeta_{2 n+1}},
$$

with $\zeta_{2 n+1}=2 n / 3+1$. Theoretical, experimental, and numerical studies support the hypothesis that the exponents $\zeta_{n}$ are anomalous, i.e., they differ from those implied by dimensional arguments in Eqs. (9) and (10) [1,2].

Structure functions for the passive scalar have been analyzed in the turbulent mixing layer in a range of scales extending from a few Kolmogorov scales up to scales as large as the mixing layer vorticity thickness. As already observed for the second-order structure function, the Reynolds

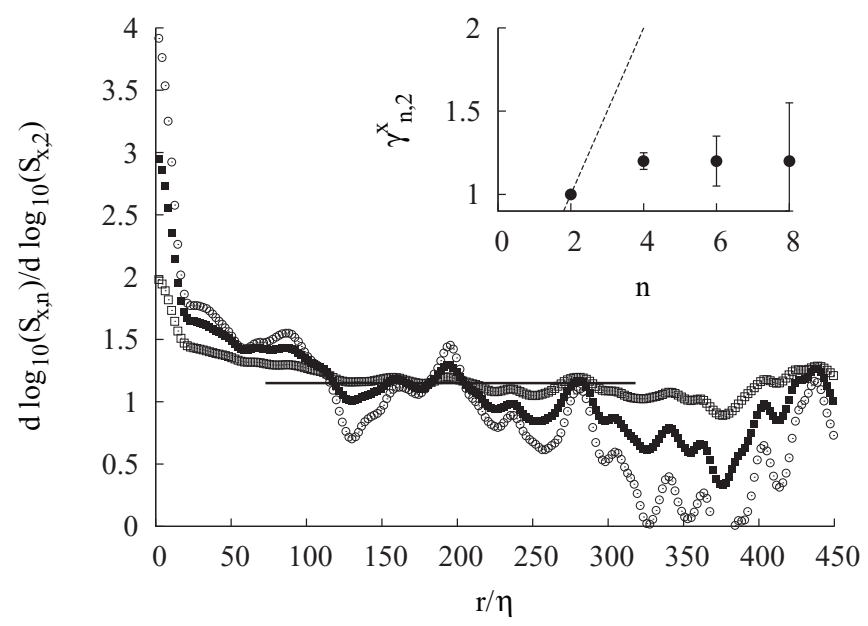

FIG. 5. Scaling of passive scalar structure functions for increments in the streamwise direction $x$. The relative logarithmic derivative $d \log _{10} S_{x, n}(r) / d \log _{10} S_{x, 2}(r)$ of structure functions of order 4 (open squares), 6 (closed squares), and 8 (open circles) is shown. The relative logarithmic derivatives are approximately constant over the range $120 \eta<r<280 \eta$. The inset shows values of the scaling exponents, evaluated as the average of the logarithmic derivatives over the range $120 \eta<r<280 \eta$. Error bars are estimated from the fluctuations of the logarithmic derivatives with respect to the average over the range $120 \eta<r<280 \eta$. The dashed line in the inset is the Kolmogorov-Obukhov-Corrsin dimensional scaling [22,23]. The horizontal line in the main figure indicates the asymptotic value $\gamma_{\infty, 2}=1.2$. 
number achieved in our simulation is not high enough to observe a well-defined inertial range that allows calculation of reliable scaling exponents. In flows at moderate Reynolds number, the extended self-similarity (ESS) concept introduced by Benzi et al. [52] has been widely applied in analyzing the scaling properties of turbulence [53-56]. The ESS concept relies on analyzing $S_{n}(r)$ as a function of $S_{2}(r)$ or $S_{3}(r)$ rather than $S_{n}(r)$ as a function of $r$. In the present work the relative exponents are computed using the second-order structure function: $\gamma_{n, 2}=\zeta_{n} / \zeta_{2}=$ $d \log _{10} S_{n}(r) / d \log _{10} S_{2}(r)$.

The logarithmic derivative $d \log _{10} S_{n}(r) / d \log _{10} S_{2}(r)$ for structure functions of order 4, 6, and 8 is shown in Fig. 5 for spatial separations $r$ in the streamwise direction $x$. The ESS slopes display a wide plateau in the range of scales between $120 \eta$ and $280 \eta$. In this scaling range, the exponent is the same for all the structure functions of order greater than 4 . This behavior is referred to as saturation of intermittency [28]. The asymptotic value of the relative exponent is approximately $\gamma_{\infty, 2}=1.2$. Assuming the spectrum scaling is $k_{x}^{-4 / 3}$, so that the second-order structure function scales as $r_{x}^{\zeta_{2}}$ with $\zeta_{2}=1 / 3$, one obtains $\zeta_{\infty}=0.4$ from $\gamma_{n, 2}=\zeta_{n} / \zeta_{2}$.

The results for separations in the spanwise direction $z$ are shown in Fig. 6. As shown in the inset, the scaling exponents are in agreement with recent results reported by Gotoh et al. [57] for a DNS of passive scalar mixing in homogeneous isotropic turbulence with a mean scalar gradient. For separations in the spanwise direction, the exponent does

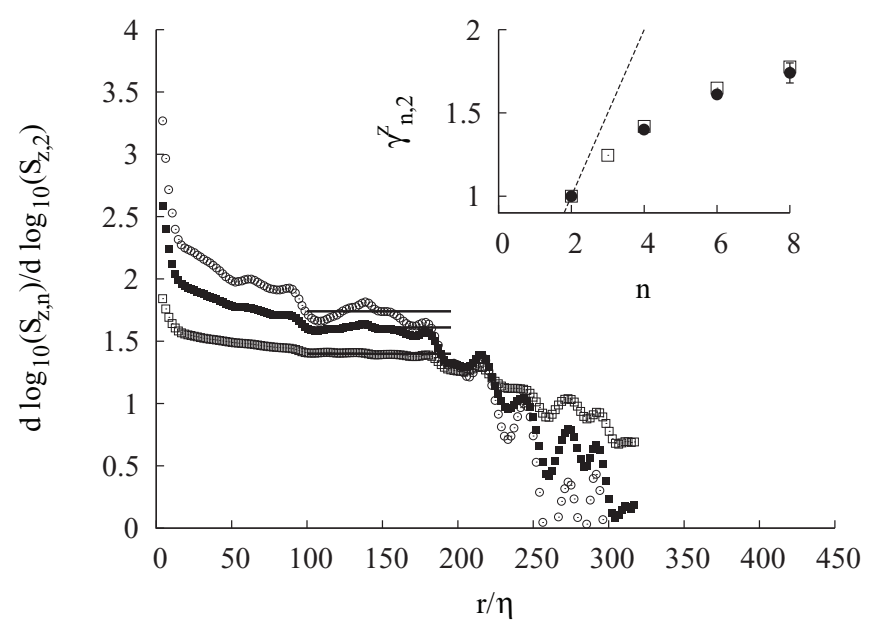

FIG. 6. Scaling of passive scalar structure functions for increments in the spanwise direction $z$. The relative logarithmic derivative $d \log _{10} S_{z, n}(r) / d \log _{10} S_{z, 2}(r)$ of structure functions of order 4 (open squares), 6 (closed squares), and 8 (open circles) is shown. The horizontal lines identify the plateaus characterizing constant scaling ranges. The inset shows values of the scaling exponents (closed symbols), evaluated as the average of the logarithmic derivatives over the range $100 \eta<r<200 \eta$, and scaling exponents from the DNS data by Gotoh et al. [57] for homogeneous isotropic turbulence advecting a passive scalar injected using a mean gradient (open symbols). Error bars are estimated from the fluctuations of the logarithmic derivatives with respect to the averages over the range $100 \eta<r<200 \eta$. The dashed line in the inset is the KolmogorovObukhov-Corrsin dimensional scaling [22,23].
TABLE II. Relative scaling exponents computed using the ESS approach [52]. The exponents are computed as the average of the logarithmic derivatives over the range indicated. The error is estimated using the maximum deviation from the average. The scaling ranges have been defined from the scaling of the second-order structure function (see the discussion in Sec. III).

\begin{tabular}{|c|c|c|}
\hline Order & $\begin{array}{c}\gamma_{n, 2}^{x} \\
120 \eta<r<280 \eta\end{array}$ & $\begin{array}{c}\gamma_{n, 2}^{z} \\
100 \eta<r<200 \eta\end{array}$ \\
\hline 2 & 1 & 1 \\
\hline 4 & $1.2 \pm 0.05$ & $1.4 \pm 0.007$ \\
\hline 6 & $1.2 \pm 0.15$ & $1.61 \pm 0.02$ \\
\hline 8 & $1.2 \pm 0.35$ & $1.74 \pm 0.06$ \\
\hline
\end{tabular}

not saturate for the orders analyzed. The values of the relative scaling exponents are summarize in Table II.

From the theoretical point of view, the existence of intermittency saturation in the passive scalar field has been proved by Balkovsky and Lebedev [58] in the idealized case of the Kraichnan model in high spatial dimensions. Celani et al. $[28,29]$ observed intermittency saturation in two-dimensional turbulence with an asymptotic exponent $\zeta_{\infty}=1.4$ and in numerical simulations of the Kraichnan model [59] with $\zeta_{\infty}=0.2$. In the Kraichnan model, saturation was observed for a roughness exponent $\gamma=0.125$ at order $n \geqslant 4$, while for larger roughness exponent values, saturation was not observed up to order 6 and higher-order statistics were not reported. For a two-dimensional Boussinesq model of thermal convection, Celani et al. [60] show saturation with $\zeta_{\infty}=0.8$. In a more realistic configuration, Antonelli et al. [32] and Mazzitelli and Lanotte [33] observe saturation of intermittency with $\zeta_{\infty}=0.6$ in large eddy simulations of convective boundary layers.

Kolmogorov's theory [61-63] and its extension to the passive scalar [22,23] invoke global scale invariance in the inertial range to describe the statistics of fluctuations. For the passive scalar field, this hypothesis implies that the scalar is scale invariant with exponent $1 / 3$, i.e., $\delta_{r} \phi \sim r^{1 / 3}$, and that the structure functions $S_{n}$ are power laws $r^{\zeta_{n}}$ with $\zeta_{n}=n / 3$. It is well known that the hypothesis of global scale invariance does not hold for three-dimensional turbulent velocity fields [48] and for the passive scalar [1], even in the simplified case of a Gaussian, nonintermittent, advecting velocity field [27,59,64]. Power laws for the structure functions are still observed, but the scaling exponents $\zeta_{n}$ deviates from the linear behavior $\zeta_{n}=n / 3$ (anomalous scaling). The limiting behavior is the saturation of intermittency, i.e., a constant value of $\zeta_{n}=\zeta_{\infty}$ at large $n$ (it is possible to show, using the maximum principle for the advection-diffusion equation, that $\zeta_{n}$ cannot decrease as $n$ increases [48]). Global scale invariance implies that the PDF of the scalar increment at different scales $r$ admits a universal form $P\left(\delta_{r} \phi\right)=r^{-1 / 3} \hat{P}\left(\delta_{r} \phi / r^{1 / 3}\right)$, where $\hat{P}$ does not depend on the separation $r$. This rescaling does not apply in the anomalous scaling case. However, in the limit of intermittency saturation, the PDF still admits a simple rescaling, but restricted to the tails $(\kappa>1)$ :

$$
P\left(\delta_{r} \phi / \sigma\right)=r^{\zeta \infty} \mathcal{P}\left(\delta_{r} \phi / \sigma\right) \quad \text { for } \quad\left|\delta_{r} \phi / \sigma\right|>\kappa \quad(\kappa>1),
$$



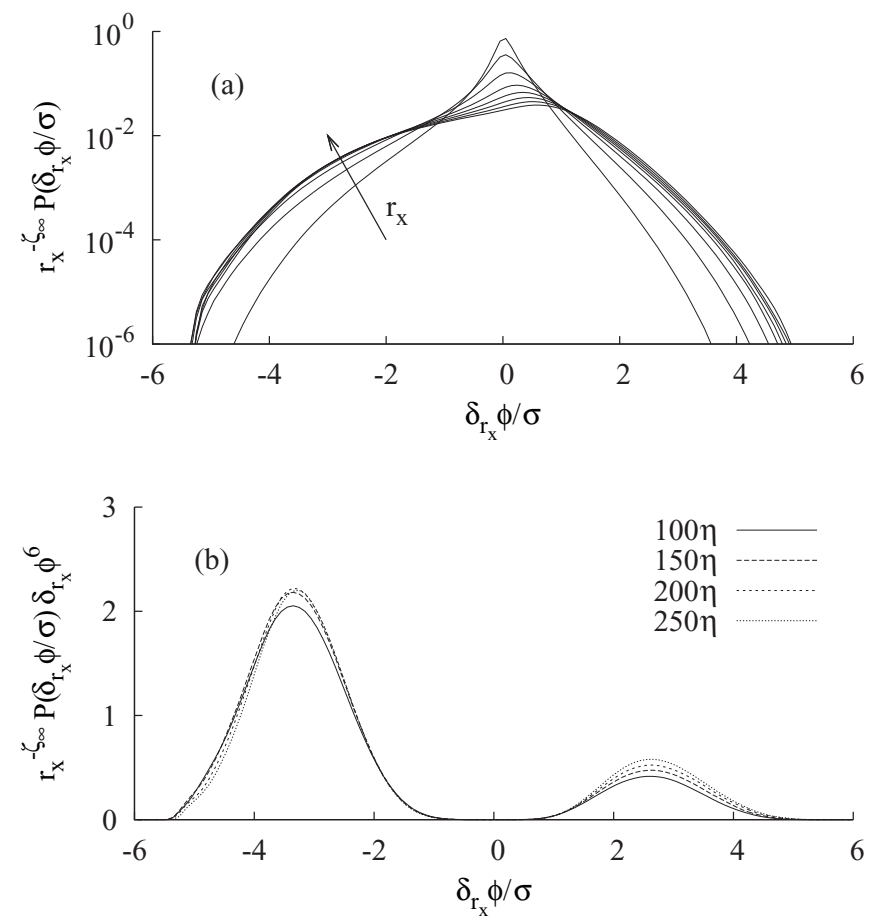

FIG. 7. (a) Probability density functions of the scalar increments $\delta \phi$ for values of the separation $r_{x}$ between $10 \eta$ and $300 \eta$, rescaled with the factor $r_{x}^{-\zeta_{\infty}}$. The arrow indicates increasing $r_{x}$. (b) Contribution of the PDFs to the sixth-order moment (the sixth-order structure function) for different separations. In the range of scale $120 \eta<r<$ $280 \eta$, the relative scaling exponents of structure functions saturate to a constant value (Fig. 5). The value $\zeta_{\infty}$ is 0.4 .

where $\mathcal{P}$ is some function that does not depend on the separation $r$ and $\sigma$ is the rms of the scalar field.

Figure 7(a) shows the PDFs of the scalar increments in the streamwise direction for separations between $10 \eta$ and $300 \eta$. Probability density functions are rescaled with $r_{x}^{-\zeta_{\infty}}$, where the value of the exponent extrapolated from the ESS scaling of the structure functions is used, $\zeta_{\infty}=0.4$. In the limit of intermittency saturation, the tails of the rescaled PDFs should collapse on the function $\mathcal{P}$ defined in Eq. (11). This property is satisfied by negative scalar increments, while the collapse is not recovered for positive increments. In order to analyze the lack of symmetry of the distribution, the contribution to the moments (i.e., the structure functions) of increments of different magnitude $P\left(\delta_{r_{x}} \phi / \sigma\right) \delta_{r_{x}} \phi^{n}$ is considered for different separations. The results are shown in Fig. 7(b) for the sixthorder moment (again rescaled by $r_{x}^{-\zeta_{\infty}}$ ). It can be observed that the major contribution to the moments comes from negative increments showing remarkable scale-independent behavior. On the contrary, the collapse is less evident for positive increments. Nonetheless, the contribution of positive scalar increments to the moment is less significant. The same behavior has been observed for all the moment orders analyzed: As the order increases, so does the difference between the two peaks; therefore the contribution of positive increments becomes less significant. The scale independence of negative increments under the rescaling in Eq. (11) and the dominant role played by the negative increments as the moment order

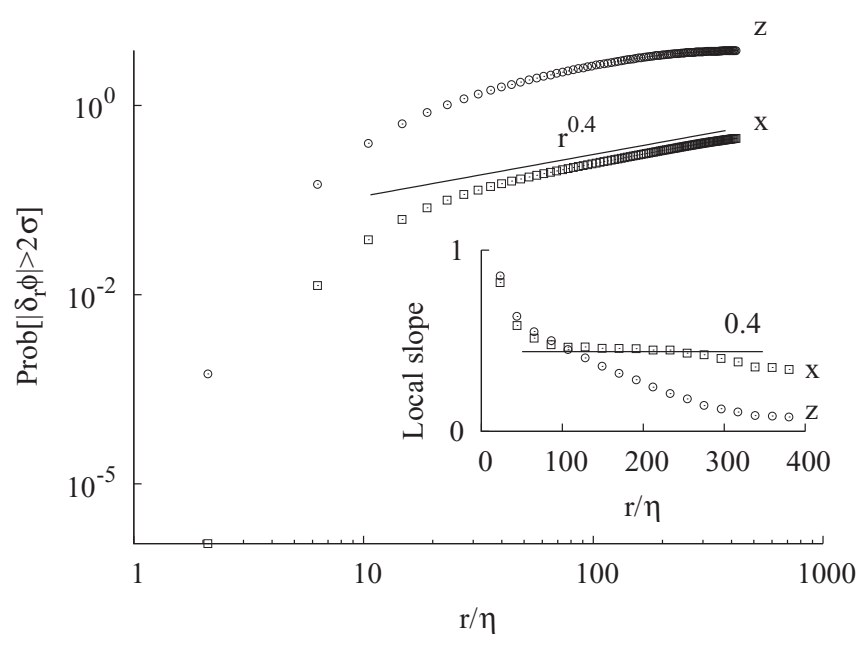

FIG. 8. Cumulative probability of having $\left|\delta_{r} \phi\right|>\kappa \sigma$, with $\kappa=2$, for increments in the streamwise $x$ (squares) and spanwise $z$ (circles) directions. The result for the spanwise direction is shifted in the vertical direction by one decade to improve readability. The inset shows the local slopes, calculated as logarithmic derivatives.

increases explain the occurrence of intermittency saturation shown in Fig. 5. The same analysis in the spanwise direction $z$ does not show the existence of an exponent $\zeta_{\infty}$, making it possible to collapse different PDFs satisfactorily on the same curve using Eq. (11).

Figure 7(b) provides an indication of the convergence of the statistics. The bulk contribution to the moment $P\left(\delta_{r_{x}} \phi / \sigma\right) \delta_{r_{x}} \phi^{6}$ decays before the tails of the PDF become noisy. The same behavior was observed for the bulk contribution to the moment of order 8 .

In addition to the direct calculation of the scaling exponents and the analysis of the tails of the PDFs, intermittency saturation can manifest in the behavior of the cumulative probability of large scalar increments [28,29,33]. Integrating Eq. (11) in the range $(-\infty,-\kappa \sigma) \cup(\kappa \sigma, \infty)$ with $\kappa>1$, the scaling $\operatorname{Prob}\left[\left|\delta_{r} \phi\right|>\kappa \sigma\right] \propto r^{\zeta_{\infty}}$ for the cumulative probability of large fluctuation is obtained. Figure 8 shows the cumulative probability of scalar increment larger than $2 \sigma$. The result is shown for separations in the streamwise and spanwise directions. A scaling range with constant slope is evident in the streamwise direction for scales $120 \eta<r<280 \eta$. In the spanwise direction there is no evidence of a plateau in the slope. Similar results have been obtained for different values of $\kappa$ in the range $(1.5,3]$. These observations confirm that intermittency saturation is present for increments in the streamwise direction and it is absent for increments in the spanwise direction.

Celani et al. [28,29,60] linked the asymptotic exponent $\zeta_{\infty}$ to the geometry of frontlike structures. The asymptotic exponent is related to the fractal dimension $D_{F}$ of the set constructed as the union of all the points where the scalar difference across a very small separation (of the order of the Kolmogorov's scale $\eta$ ) is very large (of the order of the rms $\sigma): D_{F}=d-\zeta_{\infty}(d=3$ in three dimensions). Using a box covering algorithm [65], the set of points where the scalar difference, across a length $\eta$, exceeds $\kappa \sigma(\kappa=2)$ is found to 


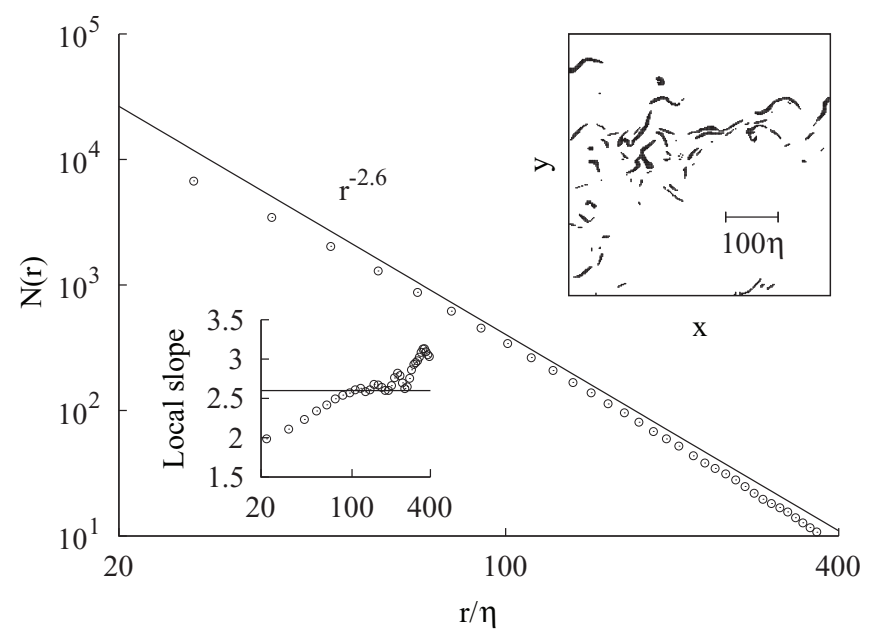

FIG. 9. Results obtained from the application of a box covering algorithm to the set of points characterized by fluctuations larger than $2 \sigma$. The number of boxes needed to cover the set $N(r)$ is plotted as a function of the size of the box $r$ (circles). The scaling of $N(r)$ versus $r$ is the fractal dimension of the set and it is close to the value $D_{F}=2.6$, in agreement with the value of the asymptotic exponent $\zeta_{\infty}=0.4$. The bottom inset shows the absolute value of the local slope (circles), calculated as a logarithmic derivative, corresponding to the fractal dimension of the set. The solid line marks the value 2.6. The top inset shows an example of an instantaneous, two-dimensional slice of the set of points where fluctuations are larger than $2 \sigma$ in the region of the mixing layer considered for the analysis.

have a fractal dimension $D_{F}=2.6$. This value agrees with the value of the asymptotic exponent $\zeta_{\infty}=0.4$ (see Fig. 9). The same result was obtained for different values of $\kappa$ in the range $(1.5,3]$.

As shown in Fig. 7, the PDFs of the scalar increments in the streamwise direction are highly asymmetric. Sreenivasan et al. [66] and Onorato and Iuso [67] investigated the asymmetry of the velocity increment PDFs by means of the plus and minus structure functions. For scalar increments in the streamwise direction, these are defined as $S_{x, n}^{ \pm}=\left\langle\left[\left(\left|\delta_{r_{x}} \phi\right| \pm \delta_{r_{x}} \phi\right) / 2\right]^{n}\right\rangle$ and characterize the scaling of the left and right sides of the PDFs. In the mixing layer, the plus and minus structure functions for increments in the spanwise direction coincide with the total structure function. Figure 10 shows a comparison of the relative scaling exponents of the total, plus, and minus structure functions in the streamwise direction and the total structure function in the spanwise direction. The scaling exponents of the minus and the total structure functions in the streamwise direction are the same, while the scaling exponent of the plus structure function in the streamwise direction is very close to that for the total structure function in the spanwise direction. The same result is observed also for structure functions of order 8 (not shown). This analysis confirms that the saturation of intermittency observed for increments in the streamwise direction is due to the negative tails of the PDFs shown in Fig. 7.

In order to characterize anisotropy at different scales, PDFs for the scalar increments in the three directions have been calculated and are shown in Fig. 11. Increments are normalized with the rms of the distribution at the specific separation;

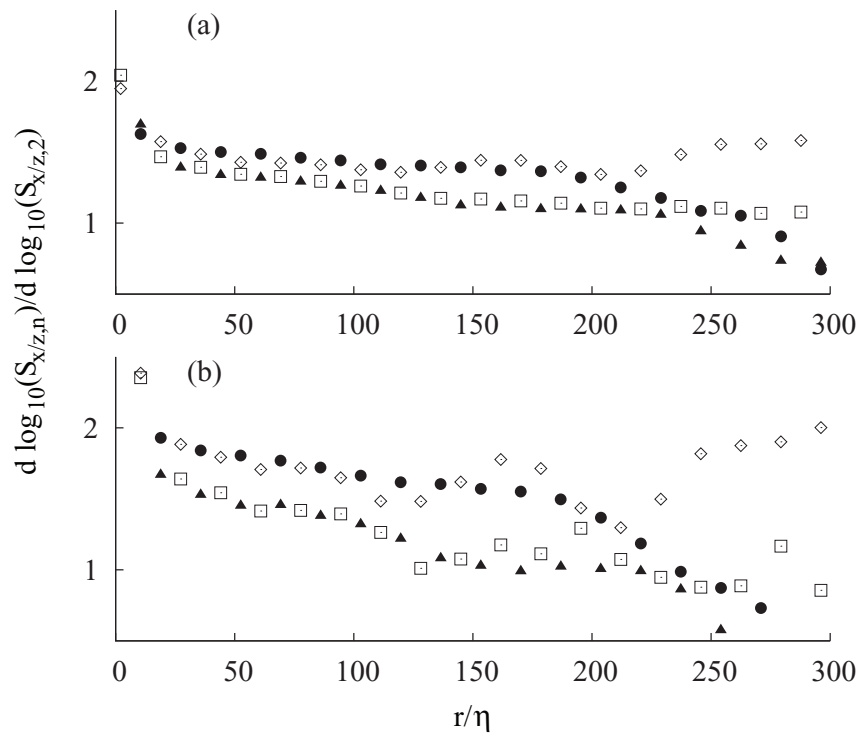

FIG. 10. Relative logarithmic derivative of the total (open squares), plus (open diamonds), and minus (closed triangles) structure functions for streamwise increments and of the total structure function for spanwise increments (closed circles). The results are shown for structure functions of order (a) 4 and (b) 6 .

therefore all curves have zero mean and unit variance. The size of the subdomain analyzed makes it possible to calculate scalar increments in the crosswise direction only across separations $r_{y}<100 \eta$; therefore PDFs in the crosswise direction are shown only up to this limit. For all three directions, the PDFs have long exponential tails at small separations and show a tendency to converge to a Gaussian shape at larger separations. The distributions in the $x$ and $y$ directions are similar and show high asymmetry while, as expected, the PDFs are symmetric in the $z$ direction.

Similar results for PDFs of scalar increments are described by Ferchichi and Tavoularis [68] for a set of measurements of a passive scalar, in homogeneous shear turbulence with mean scalar gradient. Highly asymmetric PDFs are reported in the streamwise and transverse directions with respect to the mean shear and scalar gradient. Measurements in the spanwise direction were not performed.

Intermittency saturation is usually related to the presence of frontlike structures. The similarity between PDFs in the $x$ and $y$ directions can be explained by the fact that these fronts are approximately aligned at $45^{\circ}$ with respect to the $x$ axis. Finally, intermittency saturation is not observed for the spanwise increments and the scaling exponents are indeed very similar to those obtained in homogeneous isotropic turbulence with mean scalar gradient [57]. Therefore, the signature of these structures is less evident in the $z$ direction. It is well known that the mixing layer is characterized by the quasi-two-dimensional large-scale structures observed by Brown and Roshko [6]. These structures exist in the present configuration, even in the fully turbulent far field [8]. It is likely that the geometrical structure of the passive scalar and resulting statistics of the scalar increments are closely related to these two-dimensional large structures. 

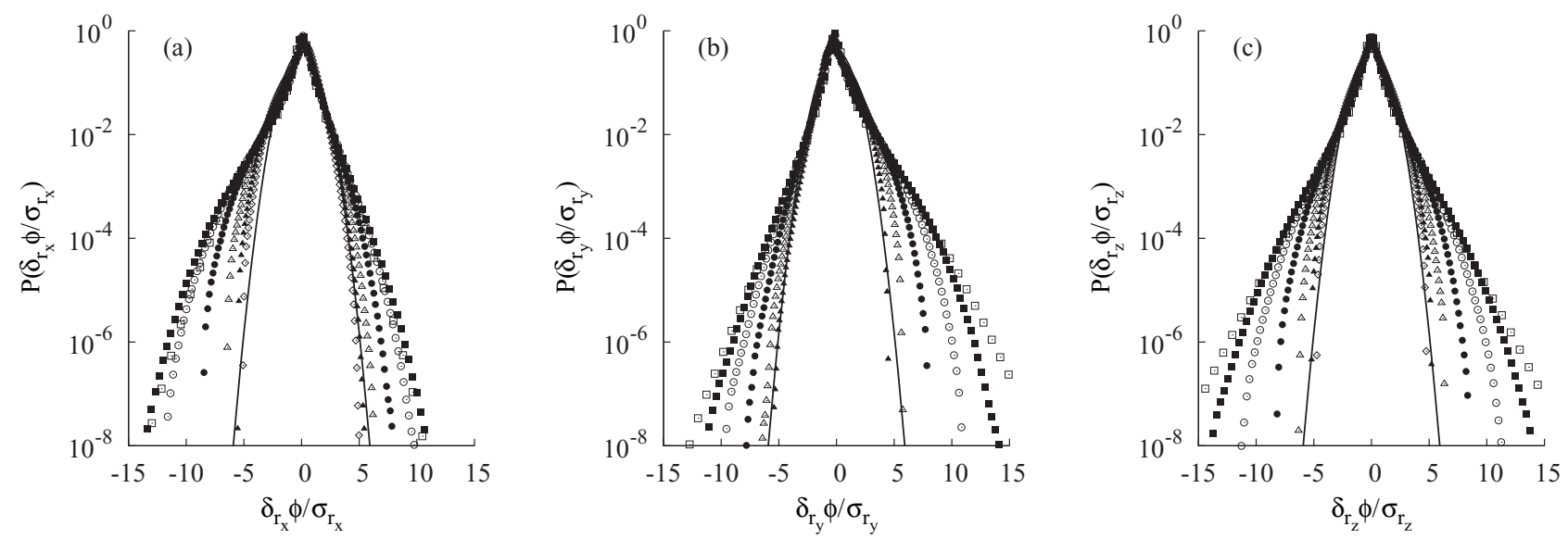

FIG. 11. Probability density functions of scalar increments for separation in the three directions: (a) streamwise $r_{x}$, (b) crosswise $r_{y}$, and (c) spanwise $r_{z}$. The statistics are shown for different separation distances: $2 \eta$ (open squares), $5 \eta$ (closed squares), 10 $\eta$ (open circles), $20 \eta$ (closed circles), $50 \eta$ (open triangles), $100 \eta$ (closed triangles), and 150 $\eta$ (open diamonds) (not shown for $y$ separation). All increments are normalized with the rms at the specific separation so that all curves have zero mean and unit variance. The solid lines represent a Gaussian distribution with zero mean and unit variance.

The deviation of the statistics of scalar increments from Gaussian behavior is summarized in Fig. 12. The skewness and flatness of the scalar increments at different separation distances are shown. The flatness peaks at around $5 \eta$ for the streamwise and crosswise directions, while for the spanwise direction it decreases monotonically and converges to the Gaussian value 3. As expected, the skewness for spanwise increments is zero. The skewness for increments in the crosswise direction is always smaller than that in the streamwise direction and peaks at $5 \eta$, while for streamwise increments it is monotonic. Finally, the absolute value of the skewness decreases as the separation distance increases.

\section{CONCLUSION}

A detailed statistical analysis of passive scalar fluctuations in a turbulent mixing layer has been performed using DNS results. The analysis has been conducted in the fully turbulent

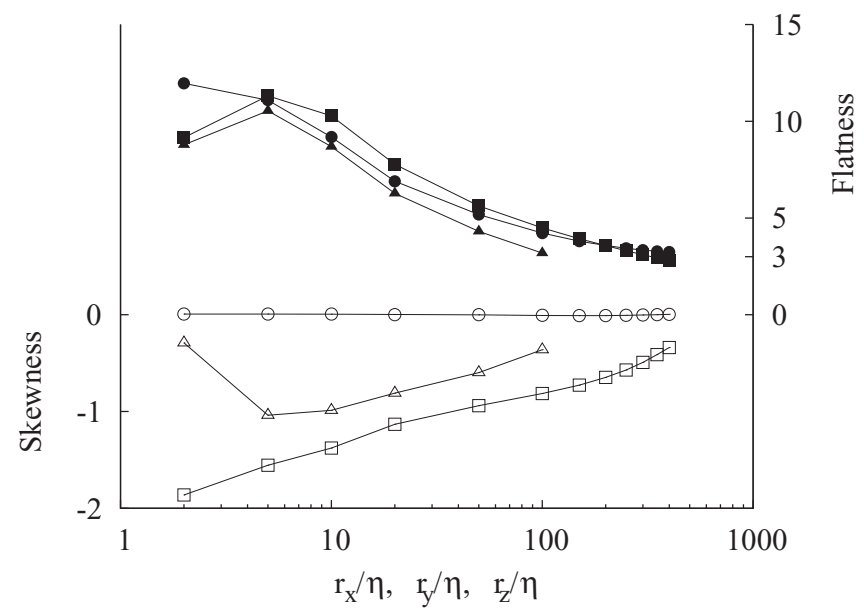

FIG. 12. Skewness (open symbols) and flatness (closed symbols) of the scalar differences for different separations in the three directions: $x$ (squares), $y$ (triangles), and $z$ (circles). far field region, where the Reynolds number based on the Taylor length scale achieves the value $\operatorname{Re}_{\lambda} \approx 250$. While the velocity spectrum shows an inertial range characterized by a scaling exponent close to $-5 / 3$ [8], for the passive scalar spectra more appropriate scaling exponents appear to be $-4 / 3$ and $-3 / 2$ in the streamwise and spanwise directions, respectively. These values are in agreement with the theoretical analysis by Celani et al. [37] for flows with mean shear. Although not wide, the inertial range of the second-order structure functions shows a scaling compatible with the spectra. The analysis of high-order statistics using the extended self-similarity concept shows that the scaling exponents of structure functions for passive scalar increments in the streamwise direction saturate to a constant value $\zeta_{\infty} \approx 0.4$ for large-order $n$ (saturation of intermittency). For the spanwise direction, saturation is not observed, at least up to structure functions of order 8 . Nonetheless, relative scaling exponents agree with recent DNS results for homogeneous isotropic turbulence with mean scalar gradient [57]. Saturation of intermittency for increments in the streamwise direction is confirmed by the collapse of the tails of the PDF under rescaling by the factor $r^{-\zeta_{\infty}}$ and by the scaling of the cumulative probability of large fluctuations. The fractal dimension of the scalar fronts is coherent with the asymptotic scaling exponent of the high-order structure function in the streamwise direction. Based on the analysis of PDFs in the three directions, it is likely that the passive scalar fronts that generate saturation of intermittency in the streamwise direction are related to the existence of the Brown and Roshko [6] structures. In contrast, these structures seem to have negligible effects on the high-order statistics in the $z$ direction.

\section{ACKNOWLEDGMENTS}

We acknowledge valuable support from KAUST Supercomputing Laboratory in the form of assistance with code development and computational time on the IBM System Blue Gene/P Shaheen at King Abdullah University of Science and Technology. 
[1] B. I. Shraiman and E. D. Siggia, Nature (London) 405, 639 (2000).

[2] Z. Warhaft, Annu. Rev. Fluid Mech. 32, 203 (2000).

[3] S. Gupta, H. G. Im, and M. Valorani, Proc. Combust. Inst. 33, 2991 (2011).

[4] K. R. Sreenivasan, Flow Turbul. Combust. 72, 115 (2004).

[5] S. Edouard, B. Legras, F. Lefevre, and R. Eymard, Nature (London) 384, 444 (1996).

[6] G. L. Brown and A. Roshko, J. Fluid Mech. 64, 775 (1974).

[7] R. D. Moser and M. M. Rogers, J. Fluid Mech. 247, 275 (1993).

[8] A. Attili and F. Bisetti, Phys. Fluids 24, 035109 (2012).

[9] A. Michalke, J. Fluid Mech. 23, 521 (1965).

[10] A. Michalke, J. Fluid Mech. 19, 543 (1964).

[11] P. E. Dimotakis, J. Fluid Mech. 409, 69 (2000).

[12] J. Bell and R. Mehta, AIAA J. 28, 2034 (1990).

[13] M. M. Rogers and R. D. Moser, J. Fluid Mech. 243, 183 (1992).

[14] R. D. Moser and M. M. Rogers, Phys. Fluids 3, 1128 (1991).

[15] M. M. Rogers and R. D. Moser, Phys. Fluids 6, 903 (1994).

[16] S. Laizet, S. Lardeau, and E. Lamballais, Phys. Fluids 22, 015104 (2010).

[17] N. D. Sandham and R. D. Sandberg, J. Turbul. 10, 1 (2009).

[18] E. Balaras, U. Piomelli, and J. M. Wallace, J. Fluid Mech. 446, 1 (2001).

[19] Y. Wang, M. Tanahashi, and T. Miyauchi, Int. J. Heat Fluid Flow 28, 1280 (2007).

[20] X. Q. Jiang, H. Gong, J. K. Liu, M. D. Zhou, and Z. S. She, J. Fluid Mech. 569, 259 (2006).

[21] Y. Tsuji and Y. Kaneda, J. Fluid Mech. 694, 50 (2012).

[22] S. Corrsin, J. Appl. Phys. 22, 469 (1951).

[23] A. M. Obukhov, Izv. Akad. Nauk SSSR Ser. Geogr. Geofiz. 13, 58 (1949).

[24] M. Holzer and E. D. Siggia, Phys. Fluids 6, 1820 (1994).

[25] M. Vergassola and A. Mazzino, Phys. Rev. Lett. 79, 1849 (1997).

[26] A. L. Fairhall, B. Galanti, V. S. L’vov, and I. Procaccia, Phys. Rev. Lett. 79, 4166 (1997).

[27] S. Chen and R. H. Kraichnan, Phys. Fluids 10, 2867 (1998).

[28] A. Celani, A. Lanotte, A. Mazzino, and M. Vergassola, Phys. Rev. Lett. 84, 2385 (2000).

[29] A. Celani, A. Lanotte, A. Mazzino, and M. Vergassola, Phys. Fluids 13, 1768 (2001).

[30] S. Kurien, K. G. Aivalis, and K. R. Sreenivasan, J. Fluid Mech. 448, 279 (2001).

[31] F. Moisy, H. Willaime, J. S. Andersen, and P. Tabeling, Phys. Rev. Lett. 86, 4827 (2001).

[32] M. Antonelli, M. Martins Afonso, A. Mazzino, and U. Rizza, J. Turbul. 6, N35 (2005).

[33] I. Mazzitelli and A. S. Lanotte, Physica D 241, 251 (2012).

[34] K. R. Sreenivasan, Proc. R. Soc. London Ser. A 434, 165 (1991).

[35] K. R. Sreenivasan, Phys. Fluids 8, 189 (1996).

[36] P. L. Miller and P. E. Dimotakis, J. Fluid Mech. 308, 129 (1996).

[37] A. Celani, M. Cencini, M. Vergassola, E. Villermaux, and D. Vincenzi, J. Fluid Mech. 523, 99 (2005).
[38] C. M. Casciola, R. Benzi, P. Gualtieri, B. Jacob, and R. Piva, Phys. Rev. E 65, 015301 (2001).

[39] O. Desjardins, G. Blanquart, G. Balarac, and H. Pitsch, J. Comput. Phys. 227, 7125 (2008).

[40] J. Kim and P. Moin, J. Comput. Phys. 59, 308 (1985).

[41] M. A. Ol'Shanskii and V. M. Staroverov, Int. J. Numer. Methods Fluid 33, 499 (2000).

[42] S. B. Pope, Turbulent Flows (Cambridge University Press, Cambridge, 2000).

[43] T. T. Clark and Y. Zhou, Phys. Rev. E 68, 066305 (2003).

[44] C. M. Casciola, P. Gualtieri, B. Jacob, and R. Piva, Phys. Rev. Lett. 95, 024503 (2005).

[45] P. Gualtieri, C. M. Casciola, R. Benzi, G. Amati, and R. Piva, Phys. Fluids 14, 583 (2002).

[46] A. Celani, M. Cencini, M. Vergassola, E. Villermaux, and D. Vincenzi, Proc. Appl. Math. Mech. 7, 1101301 (2007).

[47] G. Falkovich, K. Gawedzki, and M. Vergassola, Rev. Mod. Phys. 73, 913 (2001).

[48] U. Frisch, Turbulence: The Legacy of A. N. Kolmogorov (Cambridge University Press, Cambridge, 1995).

[49] F. Anselmet, Y. Gagne, E. J. Hopfinger, and R. A. Antonia, J. Fluid Mech. 140, 63 (1984).

[50] J. Lepore and L. Mydlarski, J. Fluid Mech. 713, 453 (2012).

[51] A. S. Monin and A. M. Yaglom, Statistical Fluid Mechanics (MIT Press, Cambridge, 1971).

[52] R. Benzi, S. Ciliberto, R. Tripiccione, C. Baudet, F. Massaioli, and S. Succi, Phys. Rev. E 48, R29 (1993).

[53] D. Segel, V. L'vov, and I. Procaccia, Phys. Rev. Lett. 76, 1828 (1996).

[54] S. Grossmann, D. Lohse, and A. Reeh, Phys. Rev. E 56, 5473 (1997).

[55] A. Arneodo, C. Baudet, F. Belin, R. Benzi, B. Castaing, B. Chabaud, R. Chavarria, S. Ciliberto, R. Camussi, F. Chilla et al., Europhys. Lett. 34, 411 (1996).

[56] G. Boffetta, A. Mazzino, S. Musacchio, and L. Vozella, Phys. Rev. E 79, 065301 (2009).

[57] T. Gotoh, T. Watanabe, and Y. Suzuki, J. Turbul. 12, N48 (2011).

[58] E. Balkovsky and V. Lebedev, Phys. Rev. E 58, 5776 (1998).

[59] R. H. Kraichnan, Phys. Fluids 11, 945 (1968).

[60] A. Celani, A. Mazzino, and M. Vergassola, Phys. Fluids 13, 2133 (2001).

[61] A. N. Kolmogorov, C. R. Acad. Sci. URSS 30, 301 (1941).

[62] A. N. Kolmogorov, C. R. Acad. Sci. URSS 31, 538 (1941).

[63] A. N. Kolmogorov, C. R. Acad. Sci. URSS 32, 16 (1941).

[64] R. H. Kraichnan, Phys. Rev. Lett. 72, 1016 (1994).

[65] K. Falconer, Fractal Geometry: Mathematical Foundations and Applications (Wiley, New York, 2007).

[66] K. R. Sreenivasan, S. I. Vainshtein, R. Bhiladvala, I. San Gil, S. Chen, and N. Cao, Phys. Rev. Lett. 77, 1488 (1996).

[67] M. Onorato and G. Iuso, Phys. Rev. E 63, 025302 (2001).

[68] M. Ferchichi and S. Tavoularis, J. Fluid Mech. 461, 155 (2002). 